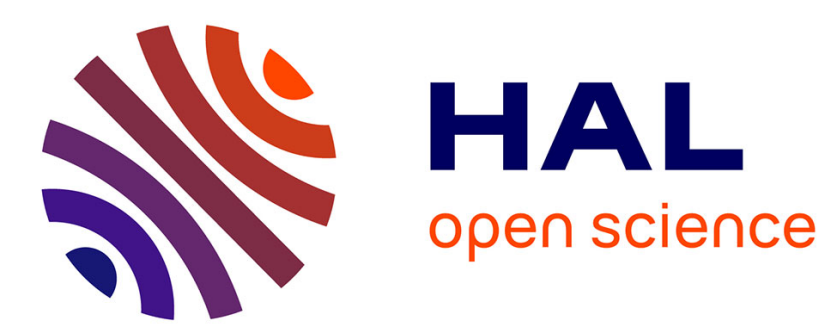

\title{
Stochastic Cramer-Rao Bound for Noncircular Signals with Application to DOA Estimation
}

\author{
Jean-Pierre Delmas, Habti Abeida
}

\section{To cite this version:}

Jean-Pierre Delmas, Habti Abeida. Stochastic Cramer-Rao Bound for Noncircular Signals with Application to DOA Estimation. IEEE Transactions on Signal Processing, 2004, 52 (11), pp.3192-3199. 10.1109/TSP.2004.836462 . hal-03399886

\section{HAL Id: hal-03399886 https://hal.science/hal-03399886}

Submitted on 24 Oct 2021

HAL is a multi-disciplinary open access archive for the deposit and dissemination of scientific research documents, whether they are published or not. The documents may come from teaching and research institutions in France or abroad, or from public or private research centers.
L'archive ouverte pluridisciplinaire HAL, est destinée au dépôt et à la diffusion de documents scientifiques de niveau recherche, publiés ou non, émanant des établissements d'enseignement et de recherche français ou étrangers, des laboratoires publics ou privés. 


\title{
Stochastic Cramer-Rao bound for non-circular signals with application to DOA estimation
}

\author{
Jean-Pierre Delmas, Habti Abeida
}

July 21, 2005

\begin{abstract}
After providing an extension of the Slepian-Bangs formula for general non-circular complex Gaussian distributions, this paper focuses on the stochastic Cramer-Rao bound (CRB) on direction of arrival (DOA) estimation accuracy for non-circular sources. We derive an explicit expression of the CRB for DOA parameters alone in the case of non-circular complex Gaussian sources by two different methods. One of them consists in computing the asymptotic covariance matrix of the maximum likelihood (ML) estimator, and the other is obtained directly from our extended Slepian-Bangs formula. Some properties of this CRB are proved and finally, it is numerically compared with the CRBs under circular complex Gaussian and complex discrete distributions of sources.
\end{abstract}

Index terms: Stochastic Cramer-Rao bound, non-circular signals, maximum likelihood estimation.

Final version T-SP01537-2003

Paper accepted to the IEEE Transactions on Signal Processing

GET/INT, Département CITI, UMR-CNRS 5157, Institut National des Télécommunications, 9 rue Charles Fourier, 91011 Evry Cedex, FRANCE. Fax: +33-1-60 764433 , e-mail: jean-pierre.delmas@int-evry.fr 


\section{Introduction}

Deterministic and stochastic CRB's play an important role in parametric estimation because the statistical performances of numerous estimation methods are known to be comparable to these bounds under certain mild conditions. Moreover, the stochastic CRB can be achieved asymptotically (in the number of measurements) by the stochastic ML method. Most of the contributions on the stochastic CRB are dedicated to Gaussian distributions for which a particularly convenient CRB formula was obtained for real Gaussian distributions by Slepian [1] and Bangs [2] referred to as the Slepian-Bangs formula, then extended to circular complex Gaussian distributions (see e.g., [3, rel. (B.3.25)]). As is well known the importance of the Gaussian CRB formulas lies in the fact that Gaussian data are rather frequently encountered in applications. Another important point is that under rather general conditions, the real [resp. circular complex] Gaussian CRB matrix is the largest of all CRB matrices among the class of arbitrary real [resp. circular complex] distributions with given mean and covariance matrices (see e.g., [3, p. 293]). However non-circular complex signals are frequently encountered in digital communications. For example, binary phase shift keying (BPSK) are often used. And no closed-form expression of the CRB is available for these signals. Consequently for non-circular complex signals, we need an upper bound of this CRB. But to the best of our knowledge, the Slepian-Bangs formula has not been yet extended to non-circular complex Gaussian distributions.

The first contribution of this paper is to give an extended Slepian-Bangs formula based on the work of [4]. Then, we concentrate on DOA estimation. For non-circular Gaussian sources, an explicit expression of the CRB for DOA parameters alone is derived from two different methods. One of them is obtained in an indirect manner

by an asymptotic analysis of the ML estimator by slight modifications of the proof given by Stoica et al [7]. And the other is obtained directly from our extended Slepian-Bangs formula by following along the lines of the paper by Stoica et al [5]. We prove that this CRB generally outperforms the circular complex Gaussian CRB associated with the same Hermitian covariance matrix. Next we prove that this CRB decreases monotonically as the noncircularity rate increases in the particular case of one source. Finally, numerical comparison of the CRB under BPSK and non-circular Gaussian distributions are given. In particular we show that for one source, the CRB under the BPSK distribution and under the non-circular complex Gaussian distribution approximately coincide. But for two equipowered uncorrelated BPSK sources, the CRB under the BPSK distribution outperforms the CRB under the non-circular complex Gaussian distribution and the difference between them is more prominent for small DOA and phase separations. 
The following notations are used throughout the paper. Matrices and vectors are represented by bold upper case and bold lower case characters, respectively. Vectors are by default in column orientation, while $T, H, *$ stand for transpose, conjugate transpose, conjugate respectively. $\odot$ is the Hadamard matrix product $\left(\right.$ i.e. $(\mathbf{A} \odot \mathbf{B}){ }_{i, j}=$ $\left.(\mathbf{A})_{i, j}(\mathbf{B})_{i, j}\right)$ and $\perp$ is the ortho-complement of a projector matrix. $\operatorname{Tr}(),. \operatorname{Det}(),. \ln (),. \Re($.$) and \Im($.$) denote the$ trace, the determinant, the logarithm, the real and the imaginary part operator, respectively.

\section{Stochastic Cramer-Rao bound for non-circular Gaussian signals}

We consider a $n$-variate complex Gaussian random variable $(\mathrm{RV}) \mathbf{z} \stackrel{\text { def }}{=} \mathbf{x}+i \mathbf{y}$, whose structured mean $\mathbf{m}_{z} \stackrel{\text { def }}{=}$ $\mathbf{m}_{x}+i \mathbf{m}_{y} \stackrel{\text { def }}{=} \mathrm{E}(\mathbf{x})+i \mathrm{E}(\mathbf{y})$ and covariance matrices $\mathbf{R}_{z} \stackrel{\text { def }}{=} \mathrm{E}\left[\left(\mathbf{z}-\mathbf{m}_{z}\right)\left(\mathbf{z}-\mathbf{m}_{z}\right)^{H}\right]$ and $\mathbf{R}_{z}^{\prime} \stackrel{\text { def }}{=} \mathrm{E}\left[\left(\mathbf{z}-\mathbf{m}_{z}\right)\left(\mathbf{z}-\mathbf{m}_{z}\right)^{T}\right]$ are parameterized by the real parameter $\Theta \in \mathcal{R}^{L}$. Considering the Fisher information matrix, we prove the following result:

Result 1 The Fisher information matrix corresponding to the non-negative definite and non-circular complex Gaussian distribution is given (elementwise) by

with

$$
\begin{array}{r}
\left(\mathbf{I}_{F}\right)_{k, l}=\left(\frac{\partial \mathbf{m}_{\tilde{z}}}{\partial \theta_{k}}\right)^{H} \mathbf{R}_{\tilde{z}}^{-1} \frac{\partial \mathbf{m}_{\tilde{z}}}{\partial \theta_{l}}+\frac{1}{2} \operatorname{Tr}\left[\frac{\partial \mathbf{R}_{\tilde{z}}}{\partial \theta_{k}} \mathbf{R}_{\tilde{z}}^{-1} \frac{\partial \mathbf{R}_{\tilde{z}}}{\partial \theta_{l}} \mathbf{R}_{\tilde{z}}^{-1}\right] \\
\mathbf{m}_{\tilde{z}} \stackrel{\text { def }}{=}\left(\begin{array}{c}
\mathbf{m}_{z} \\
\mathbf{m}_{z}^{*}
\end{array}\right) \hat{E} \text { and } \quad \mathbf{R}_{\tilde{z}} \stackrel{\text { def }}{=}\left[\begin{array}{cc}
\mathbf{R}_{z} & \mathbf{R}_{z}^{\prime} \\
\mathbf{R}_{z}^{\prime *} & \mathbf{R}_{z}^{*}
\end{array}\right] 1
\end{array}
$$

Proof: Because the non-singular $n$-variate complex Gaussian RV $\mathbf{z}$ is simply a $2 n$-variate real Gaussian RV $\left(\mathbf{x}^{T}, \mathbf{y}^{T}\right)^{T}$, with mean $\left(\mathbf{m}_{x}^{T}, \mathbf{m}_{y}^{T}\right)^{T}$ and arbitrary non-negative definite symmetric covariance matrix $\boldsymbol{\Gamma}_{2 r}$, the real Slepian-Bangs formula (see e.g., [3, rel. (B.3.3)]) can be applied:

$$
\left(\mathbf{I}_{F}\right)_{k, l}=\frac{\partial}{\partial \theta_{k}}\left(\mathbf{m}_{x}^{T}, \mathbf{m}_{y}^{T}\right) \boldsymbol{\Gamma}_{2 r}^{-1} \frac{\partial}{\partial \theta_{l}}\left(\begin{array}{c}
\mathbf{m}_{x} \\
\mathbf{m}_{y}
\end{array}\right)+\frac{1}{2} \operatorname{Tr}\left[\frac{\partial \boldsymbol{\Gamma}_{2 r}}{\partial \theta_{k}} \boldsymbol{\Gamma}_{2 r}^{-1} \frac{\partial \boldsymbol{\Gamma}_{2 r}}{\partial \theta_{l}} \boldsymbol{\Gamma}_{2 r}^{-1}\right] .
$$

\footnotetext{
${ }^{1}$ We note that contrary to $\boldsymbol{\Gamma}_{2 r}, \mathbf{R}_{\tilde{z}}$ is block structured where $\mathbf{R}_{z}$ and $\mathbf{R}_{z}^{\prime}$ are respectively Hermitian complex and symmetric complex. Consequently, the sample matrix $\mathbf{R}_{\tilde{z}, T}$ is not described by a traditional $2 n$-variate complex Wishart distribution.
} 
Then, thanks to the relation $\mathbf{R}_{\tilde{z}}=\mathbf{M} \boldsymbol{\Gamma}_{2 r} \mathbf{M}^{H}$ with $\mathbf{M} \stackrel{\text { def }}{=} \frac{1}{2}\left[\begin{array}{rc}\mathbf{I}_{n} & \mathbf{I}_{n} \\ -i \mathbf{I}_{n} & i \mathbf{I}_{n}\end{array}\right]$ proved in [4], using

$$
\left(\begin{array}{c}
\mathbf{m}_{x} \\
\mathbf{m}_{y}
\end{array}\right)=\mathbf{M}\left(\begin{array}{c}
\mathbf{m}_{z} \\
\mathbf{m}_{z}^{*}
\end{array}\right) \quad \text { and } \quad \frac{\partial \boldsymbol{\Gamma}_{2 r}}{\partial \theta_{k}} \boldsymbol{\Gamma}_{2 r}^{-1} \frac{\partial \boldsymbol{\Gamma}_{2 r}}{\partial \theta_{l}} \boldsymbol{\Gamma}_{2 r}^{-1}=\mathbf{M}^{-1} \frac{\partial \mathbf{R}_{\tilde{z}}}{\partial \theta_{k}} \mathbf{R}_{\tilde{z}}^{-1} \frac{\partial \mathbf{R}_{\tilde{z}}}{\partial \theta_{l}} \mathbf{R}_{\tilde{z}}^{-1} \mathbf{M}
$$

in (2.2), result 1 is proved.

Remark: We note that for circular complex Gaussian RVs, $\mathbf{R}_{\tilde{z}} \stackrel{\text { def }}{=}\left[\begin{array}{cc}\mathbf{R}_{z} & \mathbf{O} \\ \mathbf{O} & \mathbf{R}_{z}^{*}\end{array}\right]$; and consequently (2.1) reduces to the circular complex Gaussian Slepian-Bangs formula [3, rel. B.3.25].

\section{Application to DOA estimation for non-circular sources}

In the following, we will be concerned with the signal model

$$
\mathbf{z}_{t}=\mathbf{A} \mathbf{s}_{t}+\mathbf{n}_{t}, \quad t=1, \ldots, T
$$

where $\left(\mathbf{z}_{t}\right)_{t=1, \ldots, T}$ represents the independent identically distributed $M$-vectors of observed complex envelope at the sensor output. $\mathbf{A}=\left[\mathbf{a}_{1}, \ldots, \mathbf{a}_{K}\right]$ is the steering matrix where each vector $\mathbf{a}_{k}$ is parameterized by the real scalar parameter $\theta_{k} . \mathbf{s}_{t}=\left(s_{t, 1}, \ldots, s_{t, K}\right)^{T}$ and $\mathbf{n}_{t}$ model signals transmitted by $K$ sources and additive measurement noise respectively. $\mathbf{s}_{t}$ and $\mathbf{n}_{t}$ are multivariate independent, complex zero-mean. $\mathbf{n}_{t}$ is assumed circular complex Gaussian, spatially uncorrelated with $\mathrm{E}\left(\mathbf{n}_{t} \mathbf{n}_{t}^{H}\right)=\sigma_{n}^{2} \mathbf{I}_{M}$, while $\mathbf{s}_{t}$ is either non-circular complex Gaussian or complex discrete distributed, and possibly spatially correlated or even coherent with $\mathbf{R}_{s} \stackrel{\text { def }}{=} \mathrm{E}\left(\mathbf{s}_{t} \mathbf{s}_{t}^{H}\right)$ and $\mathbf{R}_{s}^{\prime} \stackrel{\text { def }}{=} \mathrm{E}\left(\mathbf{s}_{t} \mathbf{s}_{t}^{T}\right)$. Consequently this leads to the covariance matrices of $\mathbf{z}_{t}$ :

$$
\mathbf{R}_{z}(\Theta)=\mathbf{A} \mathbf{R}_{s} \mathbf{A}^{H}+\sigma_{n}^{2} \mathbf{I}_{M} \quad \text { and } \quad \mathbf{R}_{z}^{\prime}(\Theta)=\mathbf{A} \mathbf{R}_{s}^{\prime} \mathbf{A}^{T}
$$

If no a priori information is available, $\left(\mathbf{R}_{z}(\Theta), \mathbf{R}_{z}^{\prime}(\Theta)\right)$ is generically parametrized by the $L=K+$ $K^{2}+K(K+1)+1$ real parameters $\Theta=\left(\Theta_{1}^{T}, \Theta_{2}^{T}\right)^{T}$ with $\Theta_{1} \stackrel{\text { def }}{=}\left(\theta_{1}, \ldots, \theta_{K}\right)^{T}$ and $\Theta_{2} \stackrel{\text { def }}{=}$ $\left(\left(\Re\left(\left[\mathbf{R}_{s}\right]_{i, j}\right), \Im\left(\left[\mathbf{R}_{s}\right]_{i, j}\right), \Re\left(\left[\mathbf{R}_{s}^{\prime}\right]_{i, j}\right), \Im\left(\left[\mathbf{R}_{s}^{\prime}\right]_{i, j}\right)\right)_{1 \leq j<i \leq K},\left(\left[\mathbf{R}_{s}\right]_{i, i}, \Re\left(\left[\mathbf{R}_{s}^{\prime}\right]_{i, i}\right), \Im\left(\left[\mathbf{R}_{s}^{\prime}\right]_{i, i}\right)\right)_{i=1, \ldots, K}, \sigma_{n}^{2}\right)^{T}$. The parameter $\Theta$ is supposed identifiable from $\left(\mathbf{R}_{z}(\Theta), \mathbf{R}_{z}^{\prime}(\Theta)\right)$, in the following sense:

$$
\mathbf{R}_{z}(\Theta)=\mathbf{R}_{z}\left(\Theta^{\prime}\right) \text { and } \mathbf{R}_{z}^{\prime}(\Theta)=\mathbf{R}_{z}^{\prime}\left(\Theta^{\prime}\right) \Rightarrow \Theta=\Theta^{\prime}
$$




\subsection{Indirect derivation of the stochastic CRB for non-circular sources}

To derive the stochastic CRB of the parameter $\Theta_{1}$ alone, we consider the asymptotic covariance of the ML estimator. We first note that the probability density function (PDF) of $\mathbf{z}$ considered as a $2 M$-variate real Gaussian RV is given by an expression which is similar to that of the PDF in the circular case, provided it is expressed as a function of $\tilde{\mathbf{z}} \stackrel{\text { def }}{=}\left(\begin{array}{c}\mathbf{z} \\ \mathbf{z}^{*}\end{array}\right)$. From $[4$, rel. (15)], we have

$$
p(\mathbf{x}, \mathbf{y})=p^{\prime}(\tilde{\mathbf{z}})=(\pi)^{-M}\left[\operatorname{Det}\left(\mathbf{R}_{\tilde{z}}\right)\right]^{-1 / 2} \exp \left[-\frac{1}{2} \tilde{\mathbf{z}}^{H} \mathbf{R}_{\tilde{z}}^{-1} \tilde{\mathbf{z}}\right]
$$

where

$$
\mathbf{R}_{\tilde{z}} \stackrel{\text { def }}{=} \mathrm{E}\left(\tilde{\mathbf{z}}_{t} \tilde{\mathbf{z}}_{t}^{H}\right)=\tilde{\mathbf{A}} \mathbf{R}_{\tilde{s}} \tilde{\mathbf{A}}^{H}+\sigma_{n}^{2} \mathbf{I}_{2 M} \quad \text { with } \quad \mathbf{R}_{\tilde{s}}=\left[\begin{array}{cc}
\mathbf{R}_{s} & \mathbf{R}_{s}^{\prime} \\
\mathbf{R}_{s}^{\prime *} & \mathbf{R}_{s}^{*}
\end{array}\right]
$$

and $\tilde{\mathbf{A}} \stackrel{\text { def }}{=}\left[\begin{array}{cc}\mathbf{A} & \mathbf{O} \\ \mathbf{O} & \mathbf{A}^{*}\end{array}\right]$. Then, classically (see e.g., [6],[7]), after dropping the constants, the log-likelihood function can be written as

$$
L\left(\Theta_{1}, \Theta_{2}\right)=-\frac{T}{2}\left(\ln \left[\operatorname{Det}\left(\mathbf{R}_{\tilde{z}}\right)\right]+\operatorname{Tr}\left(\mathbf{R}_{\tilde{z}}^{-1} \mathbf{R}_{\tilde{z}, T}\right)\right)
$$

with $\mathbf{R}_{\tilde{z}, T} \stackrel{\text { def }}{=} \frac{1}{T} \sum_{t=1}^{T} \tilde{\mathbf{z}}_{t} \tilde{\mathbf{z}}_{t}^{H}$ where the parameters $\Theta_{1}$ and $\Theta_{2}$ are imbedded in the covariance matrix $\mathbf{R}_{\tilde{z}}$. In (3.4), $\mathbf{R}_{\tilde{z}}$ depends on $\mathbf{R}_{\tilde{s}}$, which is structured via (3.3). Due to these constraints, the ML estimation of $\left(\Theta_{1}, \Theta_{2}\right)$ becomes a constrained optimization problem which is not standard. Despite this difficulty, we prove in the following that the ML estimate of the DOA parameters $\Theta_{1}$ and source and noise covariance parameters $\Theta_{2}$ may be obtained in a separable form. We restrict here to the case where $K<M$ and $\mathbf{A}$ is full column rank.

Result 2 If the sample covariance matrix $\mathbf{R}_{\tilde{z}, T}$ is positive definite, the joint ML estimates which maximize the log-likelihood function (3.4) subject to the constraints (3.3) are given by the following:

$\widehat{\Theta}_{1, \mathrm{ML}}$ is obtained by the minimizing with respect to $\Theta_{1}$

$$
F_{T}\left(\Theta_{1}\right)=\ln \left[\operatorname{Det}\left(\tilde{\mathbf{A}} \widehat{\mathbf{R}}_{\tilde{s}, \mathrm{ML}} \tilde{\mathbf{A}}^{H}+\widehat{\sigma}_{n, \mathrm{ML}}^{2} \mathbf{I}_{2 M}\right)\right]
$$

where $\widehat{\mathbf{R}}_{\tilde{s}, \mathrm{ML}}$ and $\widehat{\sigma}_{n, \mathrm{ML}}^{2}$ are given by

$$
\widehat{\mathbf{R}}_{\tilde{s}, \mathrm{ML}}=\left[\tilde{\mathbf{A}}^{H}\left(\Theta_{1}\right) \tilde{\mathbf{A}}\left(\Theta_{1}\right)\right]^{-1} \tilde{\mathbf{A}}^{H}\left(\Theta_{1}\right)\left[\mathbf{R}_{\tilde{z}, T}-\widehat{\sigma}_{n, \mathrm{ML}}^{2} \mathbf{I}_{2 M}\right] \tilde{\mathbf{A}}\left(\Theta_{1}\right)\left[\tilde{\mathbf{A}}^{H}\left(\Theta_{1}\right) \tilde{\mathbf{A}}\left(\Theta_{1}\right)\right]^{-1}
$$

and

$$
\widehat{\sigma}_{n, \mathrm{ML}}^{2}=\frac{1}{M-K} \operatorname{Tr}\left(\boldsymbol{\Pi}_{\mathbf{A}\left(\Theta_{1}\right)}^{\perp} \mathbf{R}_{z, T}\right),
$$


with $\Pi_{\mathbf{A}\left(\Theta_{1}\right)}$ is the projection matrix $\mathbf{A}\left(\Theta_{1}\right)\left[\mathbf{A}^{H}\left(\Theta_{1}\right) \mathbf{A}\left(\Theta_{1}\right)\right]^{-1} \mathbf{A}^{H}\left(\Theta_{1}\right)$. Furthermore

$$
\widehat{\mathbf{R}}_{s, \mathrm{ML}}=\left[\mathbf{A}^{H}\left(\widehat{\Theta}_{1, \mathrm{ML}}\right) \mathbf{A}\left(\widehat{\Theta}_{1, \mathrm{ML}}\right)\right]^{-1} \mathbf{A}^{H}\left(\widehat{\Theta}_{1, \mathrm{ML}}\right)\left[\mathbf{R}_{z, T}-\widehat{\sigma}_{n, \mathrm{ML}}^{2} \mathbf{I}_{M}\right] \mathbf{A}\left(\widehat{\Theta}_{1, \mathrm{ML}}\right)\left[\mathbf{A}^{H}\left(\widehat{\Theta}_{1, \mathrm{ML}}\right) \mathbf{A}\left(\widehat{\Theta}_{1, \mathrm{ML}}\right)\right]^{-1}
$$

and

$$
\widehat{\mathbf{R}}_{s, \mathrm{ML}}^{\prime}=\left[\mathbf{A}^{H}\left(\widehat{\Theta}_{1, \mathrm{ML}}\right) \mathbf{A}\left(\widehat{\Theta}_{1, \mathrm{ML}}\right)\right]^{-1} \mathbf{A}^{H}\left(\widehat{\Theta}_{1, \mathrm{ML}}\right) \mathbf{R}_{z, T}^{\prime} \mathbf{A}^{*}\left(\widehat{\Theta}_{1, \mathrm{ML}}\right)\left[\mathbf{A}^{T}\left(\widehat{\Theta}_{1, \mathrm{ML}}\right) \mathbf{A}^{*}\left(\widehat{\Theta}_{1, \mathrm{ML}}\right)\right]^{-1}
$$

where $\mathbf{R}_{z, T} \stackrel{\text { def }}{=} \frac{1}{T} \sum_{t=1}^{T} \mathbf{z}_{t} \mathbf{z}_{t}^{H}$ and $\mathbf{R}_{z, T}^{\prime} \stackrel{\text { def }}{=} \frac{1}{T} \sum_{t=1}^{T} \mathbf{z}_{t} \mathbf{z}_{t}^{T}$.

Proof: Maximizing the log-likelihood (3.4) without any constraint on the Hermitian matrix $\mathbf{R}_{\tilde{s}}$ reduces to a standard maximization problem. Its solution is given (e.g., in [6], [7]) by the minimization of (3.5) where $\widehat{\mathbf{R}}_{\tilde{s}, \mathrm{ML}}$ is given by (3.6) and $\widehat{\sigma}_{n, \mathrm{ML}}^{2}$ by

$$
\widehat{\sigma}_{n, \mathrm{ML}}^{2}=\frac{1}{2 M-2 K} \operatorname{Tr}\left(\boldsymbol{\Pi}_{\tilde{\mathbf{A}}\left(\Theta_{1}\right)}^{\perp} \mathbf{R}_{\tilde{z}, T}\right) .
$$

Because $\mathbf{R}_{\tilde{z}, T}, \tilde{\mathbf{A}}^{H}\left(\Theta_{1}\right) \tilde{\mathbf{A}}\left(\Theta_{1}\right)$, then $\left[\tilde{\mathbf{A}}^{H}\left(\Theta_{1}\right) \tilde{\mathbf{A}}\left(\Theta_{1}\right)\right]^{-1}$ and $\left[\tilde{\mathbf{A}}^{H}\left(\Theta_{1}\right) \tilde{\mathbf{A}}\left(\Theta_{1}\right)\right]^{-1} \tilde{\mathbf{A}}^{H}\left(\Theta_{1}\right)$ are all partitioned of the form $\left[\begin{array}{cc}(\diamond) & (\times) \\ (\times)^{*} & (\diamond)^{*}\end{array}\right]$, the expression (3.6) is also partitioned of this form where the matrices $(\diamond)$ and $(\times)$ are given by (3.7) and (3.8) respectively. Finally, because $\boldsymbol{\Pi}_{\tilde{\mathbf{A}}\left(\Theta_{1}\right)}=\left[\begin{array}{cc}\boldsymbol{\Pi}_{\mathbf{A}\left(\Theta_{1}\right)} & \mathbf{O} \\ \mathbf{O} & \boldsymbol{\Pi}_{\mathbf{A}\left(\Theta_{1}\right)}^{*}\end{array}\right]$ and $\mathbf{R}_{\tilde{z}, T}=\left[\begin{array}{cc}\mathbf{R}_{z, T} & \mathbf{R}_{z, T}^{\prime} \\ \mathbf{R}_{z, T}^{\prime *} & \mathbf{R}_{z, T}^{*}\end{array}\right]$,
result 2 is proved.

Because the dimension of $\Theta$ that parametrizes our model is fixed, it follows from the standard statistical theory of ML estimator (see e.g., [8]) that the ML estimator of $\Theta_{1}$ asymptotically (in the number of measurements) achieves the CRB for $\Theta_{1}$ estimation. Consequently, an explicit expression of the CRB of $\Theta_{1}$ alone can be derived thanks to an asymptotic analysis of the ML estimate of $\Theta_{1}$ given by result 2. Thus, by adapting the proof given in [7], the following result is proved.

Result 3 The normalized (i.e. for $T=1$ ) DOA-related block of CRB for non-circular complex Gaussian (NCG) sources is given by the following explicit expression:

$$
\left.\mathbf{C}_{\Theta_{1}}^{\mathrm{NCG}}=\frac{\sigma_{n}^{2}}{2}\left\{\Re\left[\mathbf{D}^{H} \mathbf{\Pi}_{\mathbf{A}}^{\perp} \mathbf{D} \odot\left(\left[\mathbf{R}_{s} \mathbf{A}^{H}, \mathbf{R}_{s}^{\prime} \mathbf{A}^{T}\right] \mathbf{R}_{\tilde{z}}^{-1}\left[\begin{array}{c}
\mathbf{A} \mathbf{R}_{s} \\
\mathbf{A}^{*} \mathbf{R}_{s}^{\prime *}
\end{array}\right]\right)\right)^{T}\right]\right\}^{-1}
$$

with $\mathbf{D} \stackrel{\text { def }}{=} \frac{d \mathbf{A}\left(\Theta_{1}\right)}{d \Theta_{1}}$. 
Proof: Deriving the covariance $\mathbf{C}_{\Theta_{1}}$ of the asymptotic distribution of the minimizing $\Theta_{1, T}$ of the function $F_{T}\left(\Theta_{1}\right)$ (see (3.5)) depending on the unknown $\Theta_{1}$ and the statistics $\mathbf{R}_{\tilde{z}, T}$ is a standard problem. It solution is given by (see e.g., [5])

$$
\mathbf{C}_{\Theta_{1}}=\left[\mathbf{F}^{\prime \prime}\left(\Theta_{1}\right)\right]^{-1}\left(\lim _{T \rightarrow \infty} \mathrm{E}\left(\left[\mathbf{F}_{T}^{\prime}\left(\Theta_{1}\right)\right]\left[\mathbf{F}_{T}^{\prime}\left(\Theta_{1}\right)\right]^{T}\right)\right)\left[\mathbf{F}^{\prime \prime}\left(\Theta_{1}\right)\right]^{-1}
$$

where $\mathbf{F}_{T}^{\prime}\left(\Theta_{1}\right)$ is the gradient of $\mathbf{F}_{T}\left(\Theta_{1}\right)$ and $\mathbf{F}^{\prime \prime}\left(\Theta_{1}\right)$ is the limit of the Hessian of $\mathbf{F}_{T}\left(\Theta_{1}\right)$ when $T \rightarrow \infty$. Because, it is proved in result 2, that the constrained ML estimate of $\Theta_{1}$ (i.e. with $\mathbf{R}_{\tilde{s}}$ satisfying the constraints (3.3)) coincides with the unconstrained ML estimate of $\Theta_{1}$ (i.e. where $\mathbf{R}_{\tilde{s}}$ is an arbitrary Hermitian matrix), we can follow along the lines of the derivation given in [7] where $\mathbf{R}_{z}=\mathbf{A} \mathbf{R}_{s} \mathbf{A}^{H}+\sigma_{n}^{2} \mathbf{I}_{M}$ is replaced here by $\mathbf{R}_{\tilde{z}}=\tilde{\mathbf{A}} \mathbf{R}_{\tilde{s}} \tilde{\mathbf{A}}^{H}+\sigma_{n}^{2} \mathbf{I}_{2 M} . \mathbf{F}_{T}^{\prime}\left(\Theta_{1}\right)$ and $\mathbf{F}^{\prime \prime}\left(\Theta_{1}\right)$, then $\mathbf{C}_{\Theta_{1}}^{\mathrm{NCG}}$ are derived in Appendix A thanks to slight modifications w.r.t. [7]. More precisely, [7, rel. (3.7)] is generalized to

$$
\left(\mathbf{F}_{T}^{\prime}\left(\Theta_{1}\right)\right)_{k}=2 \Re\left[\mathbf{h}_{k, T}^{H} \mathbf{R}_{z, T} \boldsymbol{\Pi}_{\mathbf{A}}^{\perp} \mathbf{d}_{k}+\mathbf{g}_{k, T}^{H} \mathbf{R}_{z, T}^{*} \boldsymbol{\Pi}_{\mathbf{A}}^{\perp} \mathbf{d}_{k}\right], \quad k=1, \ldots, K
$$

and $[7$, rel. (3.8)] is generalized to

$$
\mathbf{F}^{\prime \prime}\left(\Theta_{1}\right)=\frac{2}{\sigma_{n}^{2}} \Re\left[\mathbf{D}^{H} \boldsymbol{\Pi}_{\mathbf{A}}^{\perp} \mathbf{D} \odot\left(\left[\mathbf{R}_{s} \mathbf{A}^{H}, \mathbf{R}_{s}^{\prime} \mathbf{A}^{T}\right] \mathbf{R}_{\tilde{z}}^{-1}\left[\begin{array}{c}
\mathbf{A} \mathbf{R}_{s} \\
\mathbf{A}^{*} \mathbf{R}_{s}^{\prime *}
\end{array}\right]\right)^{T}\right]
$$

where $\mathbf{h}_{k, T}^{H}$ and $\mathbf{g}_{k, T}^{H}$ are respectively the $k$ th row of the matrices

$$
\left[\left(\tilde{\mathbf{A}}^{H} \mathbf{R}_{\tilde{z}, T} \tilde{\mathbf{A}}\right)_{(1: K, 1: K)}^{-1}-\frac{1}{\sigma_{n}^{2}}\left(\mathbf{A}^{H} \mathbf{A}\right)^{-1}\right] \mathbf{A}^{H} \text { and }\left(\tilde{\mathbf{A}}^{H} \mathbf{R}_{\tilde{z}, T} \tilde{\mathbf{A}}\right)_{(1: K, K+1: 2 K)}^{-1} \mathbf{A}^{T}
$$

Remark 1: We note that for circular complex Gaussian (CG) sources, $\mathbf{R}_{s}^{\prime}=\mathbf{O}$ and $\mathbf{R}_{\tilde{z}}=\left[\begin{array}{cc}\mathbf{R}_{z} & \mathbf{O} \\ \mathbf{O} & \mathbf{R}_{z}^{*}\end{array}\right]$. Consequently (3.9) reduces to

$$
\mathbf{C}_{\Theta_{1}}^{\mathrm{CG}}=\frac{\sigma_{n}^{2}}{2}\left\{\Re\left[\mathbf{D}^{H} \boldsymbol{\Pi}_{\mathbf{A}}^{\perp} \mathbf{D} \odot\left(\mathbf{R}_{s} \mathbf{A}^{H} \mathbf{R}_{z}^{-1} \mathbf{A} \mathbf{R}_{s}\right)^{T}\right]\right\}^{-1}
$$

indirectly derived in [7], then directly derived from the circular complex Stepian-Bangs formula in [5].

The next result compares the CRBs $\mathbf{C}_{\Theta_{1}}^{\mathrm{NCG}}$ and $\mathbf{C}_{\Theta_{1}}^{\mathrm{CG}}$ associated with sources with the same first covariance $\operatorname{matrix} \mathbf{R}_{s}$.

Result 4 The DOA-related block of CRB for non-circular complex Gaussian sources is upper bounded by the associated CRB for circular complex Gaussian sources corresponding to the same first covariance matrix $\mathbf{R}_{s}$.

$$
\mathbf{C}_{\Theta_{1}}^{\mathrm{NCG}} \leq \mathbf{C}_{\Theta_{1}}^{\mathrm{CG}}
$$


Proof: First, from [7, lemma A.4], we have $\mathbf{B}_{1}-\mathbf{B}_{2} \geq \mathbf{O}$ with $\mathbf{B}_{1} \stackrel{\text { def }}{=}\left[\mathbf{R}_{s} \mathbf{A}^{H}, \mathbf{R}_{s}^{\prime} \mathbf{A}^{T}\right] \mathbf{R}_{\tilde{z}}^{-1}\left[\begin{array}{c}\mathbf{A} \mathbf{R}_{s} \\ \mathbf{A}^{*} \mathbf{R}_{s}^{\prime *}\end{array}\right]$ and $\mathbf{B}_{2} \stackrel{\text { def }}{=} \mathbf{R}_{s} \mathbf{A}^{H} \mathbf{R}_{z}^{-1} \mathbf{A} \mathbf{R}_{s}$ and this inequality applies to the transpose of these matrices: $\mathbf{B}_{1}^{T}-\mathbf{B}_{2}^{T} \geq \mathbf{O}$. Then, because $\mathbf{B}_{3} \stackrel{\text { def }}{=} \mathbf{D}^{H} \boldsymbol{\Pi}_{\mathbf{A}}^{\perp} \mathbf{D} \geq \mathbf{O}$, we have thanks to a standard result of linear algebra (see e.g., [3, Appendix A, result R.19], $\mathbf{B}_{3} \odot\left(\mathbf{B}_{1}^{T}-\mathbf{B}_{2}^{T}\right) \geq \mathbf{O}$. This inequality is extended to the associated real symmetric matrices $\Re\left[\mathbf{B}_{3} \odot \mathbf{B}_{1}^{T}\right]-\Re\left[\mathbf{B}_{3} \odot \mathbf{B}_{2}^{T}\right] \geq \mathbf{O}$, then by inversion $\left\{\Re\left[\mathbf{B}_{3} \odot \mathbf{B}_{1}^{T}\right]\right\}^{-1}-\left\{\Re\left[\mathbf{B}_{3} \odot \mathbf{B}_{2}^{T}\right]\right\}^{-1} \leq \mathbf{O}$.

In the particular case of one source, we prove the following:

Result 5 The CRB of $\theta_{1}$ for a non-circular complex Gaussian source decreases monotonically as the noncircularity rate increases and is given by the expression

$$
C_{\theta_{1}}^{\mathrm{NCG}}=\frac{1}{\alpha}{ }_{1}\left[\frac{2 r_{1}^{-1}+\left\|\mathbf{a}_{1}\right\|^{-2} r_{1}^{-2}+\left\|\mathbf{a}_{1}\right\|^{2}-\left\|\mathbf{a}_{1}\right\|^{2} \rho_{1}^{2}}{\left\|\mathbf{a}_{1}\right\|^{2} r_{1}+1+\left(1-\left\|\mathbf{a}_{1}\right\|^{2} r_{1}\right) \rho_{1}^{2}}\right]
$$

where the non-circularity rate $\rho_{1}$ is defined by $\mathrm{E}\left(s_{t, 1}^{2}\right)=\rho_{1} e^{i \phi_{1}} \mathrm{E}\left|s_{t, 1}^{2}\right|$ and satisfies $0 \leq \rho_{1} \leq 1$. $\phi_{1}$ is the circularity phase of $s_{t, 1}$, (it will be used in Section 3.3). The SNR is defined by $r_{1} \stackrel{\text { def }}{=} \frac{\sigma_{1}^{2}}{\sigma_{n}^{2}}$ and $\alpha_{1}$ is the purely geometrical factor $2 \mathbf{a}_{1}^{\prime}{ }^{H} \mathbf{\Pi}_{\mathbf{a}_{1}}^{\perp} \mathbf{a}_{1}^{\prime}$ with $\mathbf{a}_{1}^{\prime} \stackrel{\text { def }}{=} \frac{d \mathbf{a}_{1}}{d \theta_{1}}$.

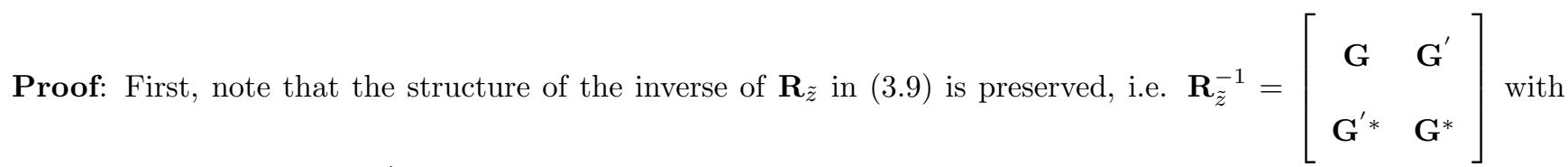
$\mathbf{G}=\left(\mathbf{R}_{z}-\mathbf{R}_{z}^{\prime} \mathbf{R}_{z}^{*-1} \mathbf{R}_{z}^{*}\right)^{-1}$ and $\mathbf{G}^{\prime}=-\mathbf{G R}_{z}^{\prime} \mathbf{R}_{z}^{*-1}$. With $\mathbf{R}_{z}=\sigma_{1}^{2} \mathbf{a}_{1} \mathbf{a}_{1}^{H}+\sigma_{n}^{2} \mathbf{I}_{M}$ and $\mathbf{R}_{z}^{\prime}=\sigma_{1}^{2} \rho_{1} e^{i \phi_{1}} \mathbf{a}_{1} \mathbf{a}_{1}^{T},(3.14)$ follows thanks to straightforward but tedious calculations. The monotony of $C_{\theta_{1}}^{\mathrm{NCG}}$ with $\rho_{1}$ is proved in Appendix B.

Consequently for one source, the CRB decreases from $C_{\theta_{1}}=\frac{1}{\alpha_{1} r_{1}}\left(1+\frac{1}{\left\|\mathbf{a}_{1}\right\|^{2} r_{1}}\right)\left(\rho_{1}=0\right.$, circular case $)$ to $C_{\theta_{1}}=\frac{1}{\alpha_{1} r_{1}}\left(1+\frac{1}{2\left\|\mathbf{a}_{1}\right\|^{2} r_{1}}\right)\left(\rho_{1}=1\right.$, unfiltered BPSK case $)$.

\subsection{Direct derivation of the stochastic CRB for non-circular sources}

To directly prove result 3 from the Fisher information matrix (2.1), we first note that thanks to the proof of result 2, the constrained ML estimate of $\Theta_{1}$ coincides with the unconstrained ML estimate of $\Theta_{1}$. Consequently the associated CRBs of $\Theta_{1}$ coincide for these two models. Using the unconstrained model, let $\Theta=\left(\Theta_{1}^{T}, \Theta_{2}^{T}\right)^{T}$ with here $\Theta_{2} \stackrel{\text { def }}{=}\left(\boldsymbol{\rho}^{T}, \sigma_{n}^{2}\right)^{T}$ where $\left.\boldsymbol{\rho} \stackrel{\text { def }}{=}\left(\left(\Re\left(\left[\mathbf{R}_{\tilde{s}}\right]_{i, j}\right), \Im\left(\left[\mathbf{R}_{\tilde{s}}\right]_{i, j}\right)\right)_{1 \leq j<i \leq 2 K},\left(\left[\mathbf{R}_{\tilde{s}}\right]_{i, i}\right)\right)_{i=1, \ldots, 2 K}\right)^{T}$. With this unconstrained 
model, we can follow along the lines of the derivation given in [5] where $\mathbf{R}_{z}=\mathbf{A} \mathbf{R}_{s} \mathbf{A}^{H}+\sigma_{n}^{2} \mathbf{I}_{M}$ is replaced here by $\mathbf{R}_{\tilde{z}}=\tilde{\mathbf{A}} \mathbf{R}_{\tilde{s}} \tilde{\mathbf{A}}^{H}+\sigma_{n}^{2} \mathbf{I}_{2 M}$ because the key point of the derivation, i.e. the relation $\operatorname{vec}\left(\mathbf{R}_{\tilde{s}}\right)=\mathbf{J} \boldsymbol{\rho}$ where $\mathbf{J}$ is a constant nonsingular complex matrix is preserved. And rel. (3.9) is proved in Appendix C thanks to slight modifications of the direct derivation given in [5].

We note that the validity conditions of result 2 are " $K<M$ and $\mathbf{A}$ is full column rank", whereas the identifiability condition (3.1) does not impose such conditions if a priori knowledge is available. For example, in the case of a uniform linear array and $K$ independent sources of maximum non-circularity rates $\left(\rho_{k}=1, k=1, \ldots, K\right)$, it is shown in the simulations of [9] that up to $K=2 M-2$ sources can be identified. In these cases, we have to resort to the CRB derived from the closed-form expression of the asymptotic minimum variance for complex non-circular Gaussian signals [11]. This remark extends to non-circular Gaussian signals, the discussion considered in [10] for circular Gaussian signals.

\subsection{Illustrative examples}

The purpose of this section is to illustrate results 3,4 and 5 and to compare these CRBs to the CRB associated with BPSK distributed sources. We consider throughout this section one or two independent and equipowered sources with identical non-circularity rate. These sources impinge on a uniform linear array of $M$ sensors separated by a half-wavelength for which $\mathbf{a}_{k}=\left(1, e^{i \theta_{k}}, \ldots, e^{i(M-1) \theta_{k}}\right)^{T}$ where $\theta_{k}=\pi \sin \left(\alpha_{k}\right)$ with $\alpha_{k}$, the DOAs relative to the normal of array broadside.

The first experiment illustrates results 3 and 4. We consider two non-circular complex Gaussian sources with $M=6$ and $S N R=20 d B$. Figs. 1,2 and 3 exhibit the dependence of $\left(\mathbf{C}_{\Theta_{1}}^{\mathrm{NCG}}\right)_{(1,1)}{ }^{2}$ with the non-circularity rate $\rho_{1}=\rho_{2}$, the circularity phase separation $\phi_{2}-\phi_{1}$ and the DOA separation $\theta_{2}-\theta_{1}$ respectively. Fig. 1 shows that $\left(\mathbf{C}_{\Theta_{1}}^{\mathrm{NCG}}\right)_{(1,1)}$ decreases as the non-circularity rate incresases (this extends to two equipowered sources result 5 proved in the one source case). Furthermore this decrease is more prominent for low DOA separations. Fig. 2 shows that $\left(\mathbf{C}_{\Theta_{1}}^{\mathrm{NCG}}\right)_{(1,1)}$ is sensitive to the circularity phase separation for low DOA separations. And Fig. 3 illustrates the inequality (3.13) of result 4. It shows that the difference between these two values is very sensitive for very low DOA separations only. Fig. 4 compares the non-circular complex Gaussian CRB $\mathbf{C}_{\Theta_{1}}^{\mathrm{NCG}}$ with the non-circular complex Gaussian $\mathrm{CRB} \mathbf{C}_{\Theta_{1}}^{\mathrm{NCG}^{\prime}}$ under the a priori information that the two sources are independent

\footnotetext{
${ }^{2}$ All the CRBs are computed for $T=1$. That means that the actual CRBs associated with the signal model defined in section 3 are obtained from the results given in this section by dividing by $T$.
} 
${ }^{3}$ given in [11] by a non-explicit expression. Fig. 4 shows that this a priori information is quite informative, but this information gain decreases as the non-circularity rate increases. This is particularly prominent for low DOA separations.

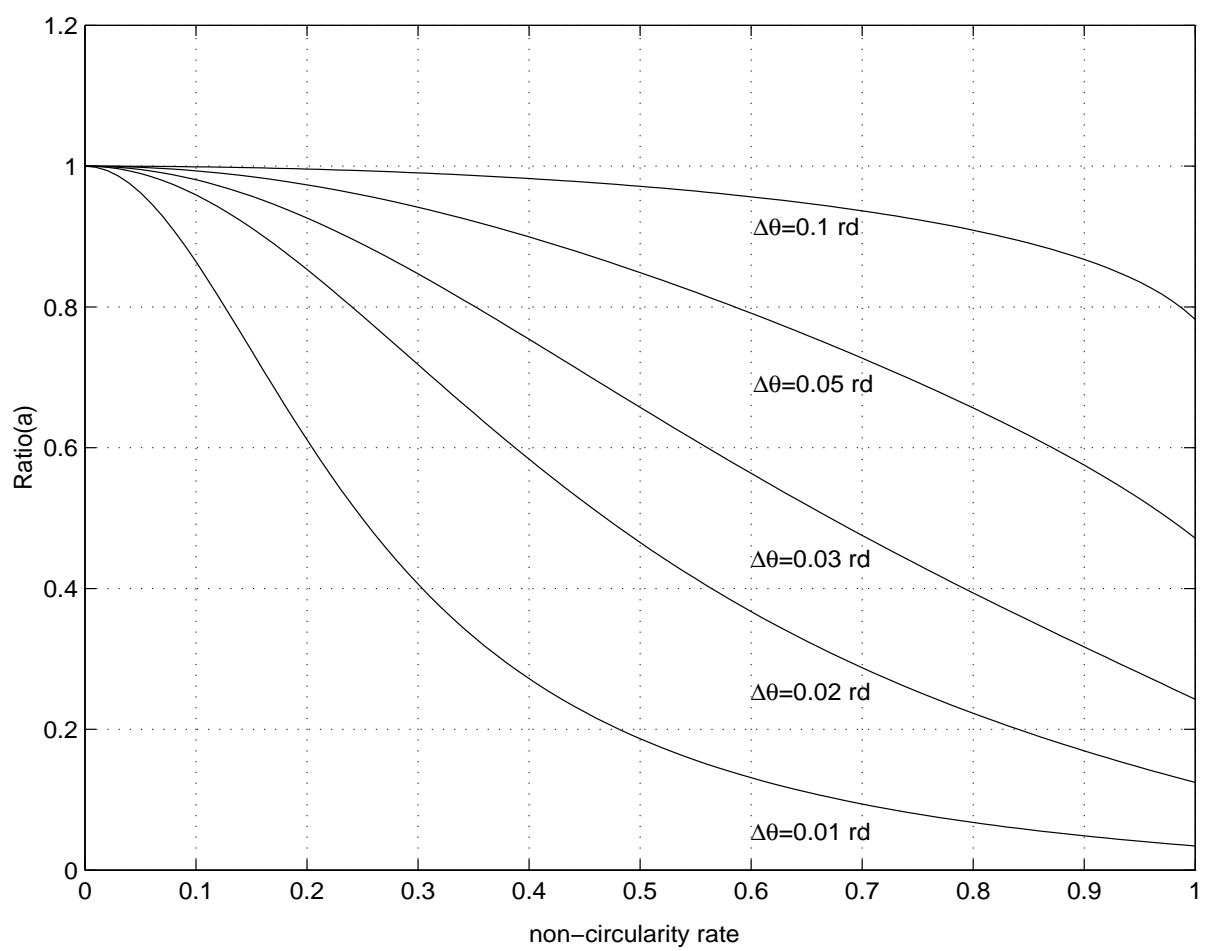

Fig.1 Ratio $r_{a} \stackrel{\text { def }}{=} \frac{\left(\mathbf{C}_{\Theta_{1}}^{\mathrm{NCG}}\right)_{(1,1)}}{\left(\mathbf{C}_{\Theta_{1}}^{\mathrm{CG}}\right)_{(1,1)}}$ as a function of the non-circularity rate for different values of DOA separation $(\Delta \theta)$ for $\phi_{1}=\pi / 2$ and $\phi_{2}=\pi / 3$.

${ }^{3}$ We note that the explicit expression (3.9) does not take account of this a priori information because it has been derived without any constraint on $\mathbf{R}_{s}$ and $\mathbf{R}_{s}^{\prime}$. 


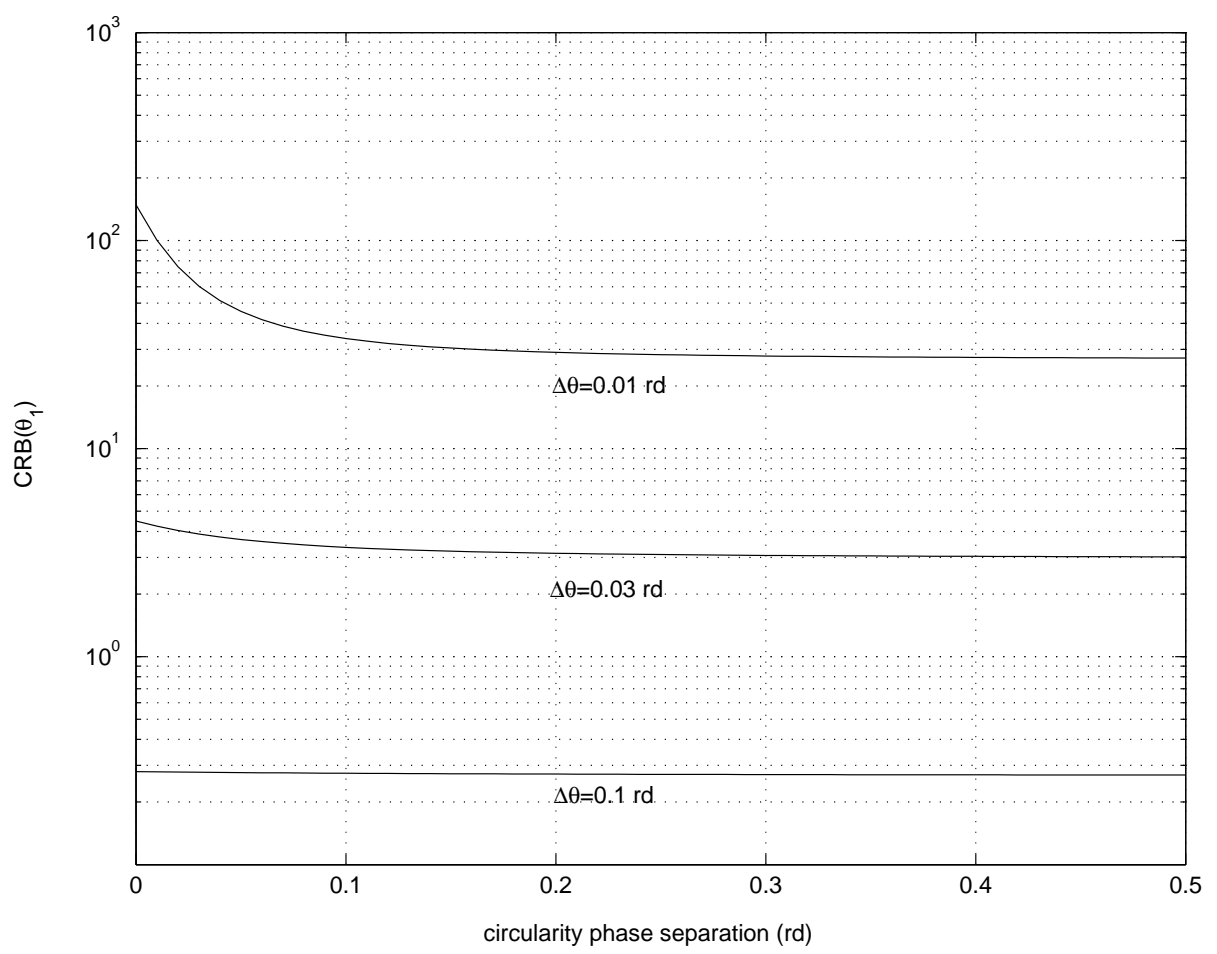

Fig.2 $\left(\mathbf{C}_{\Theta_{1}}^{\mathrm{NCG}}\right)_{(1,1)}$ as a function of the circularity phase separation for different values of DOA separation $(\Delta \theta)$ for $\rho_{1}=\rho_{2}=1$.

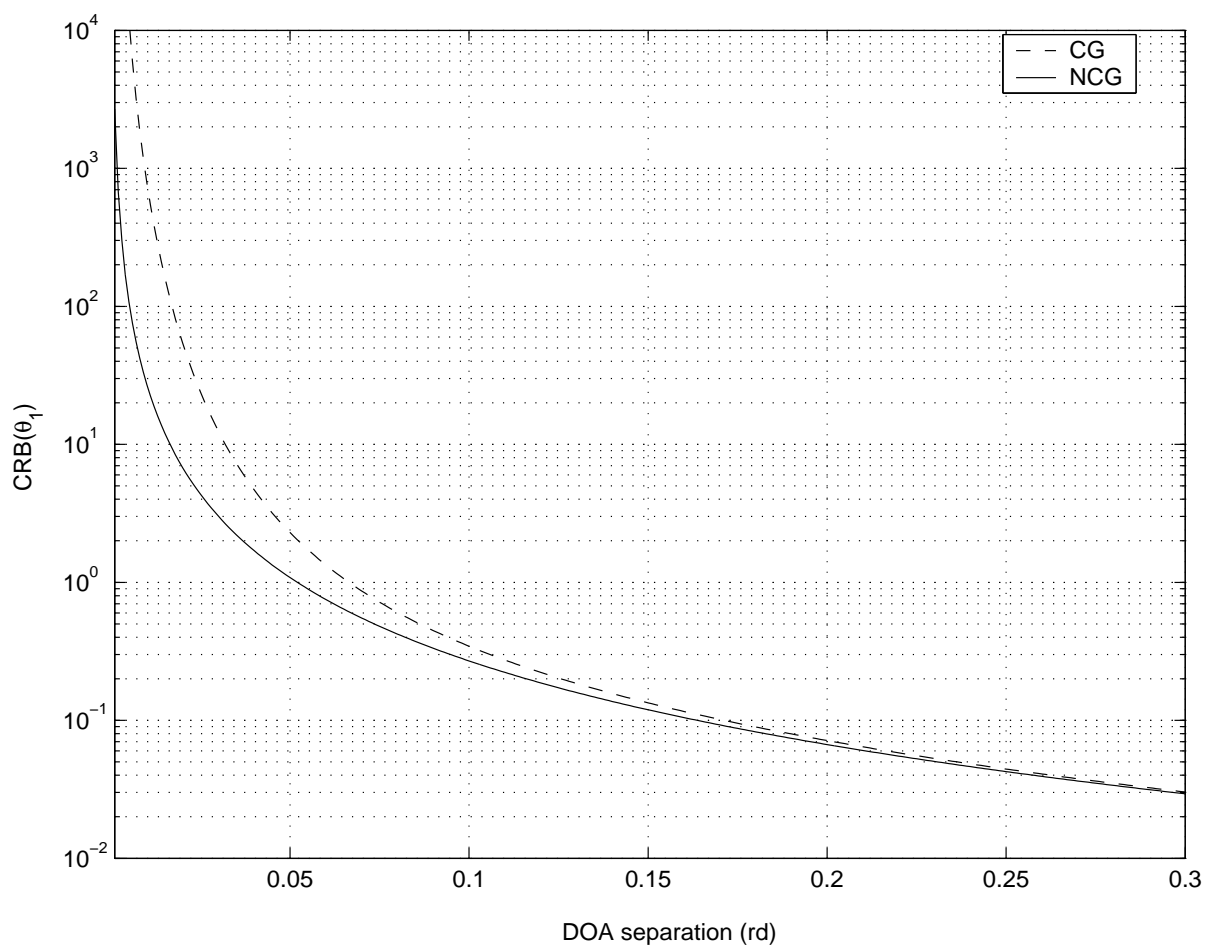

Fig.3 $\left(\mathbf{C}_{\Theta_{1}}^{\mathrm{NCG}}\right)_{(1,1)}$ and $\left(\mathbf{C}_{\Theta_{1}}^{\mathrm{CG}}\right)_{(1,1)}$ as a function of the DOA separation for $\rho_{1}=\rho_{2}=1$ and $\phi_{1}=\pi / 2$ and $\phi_{2}=\pi / 3$. 


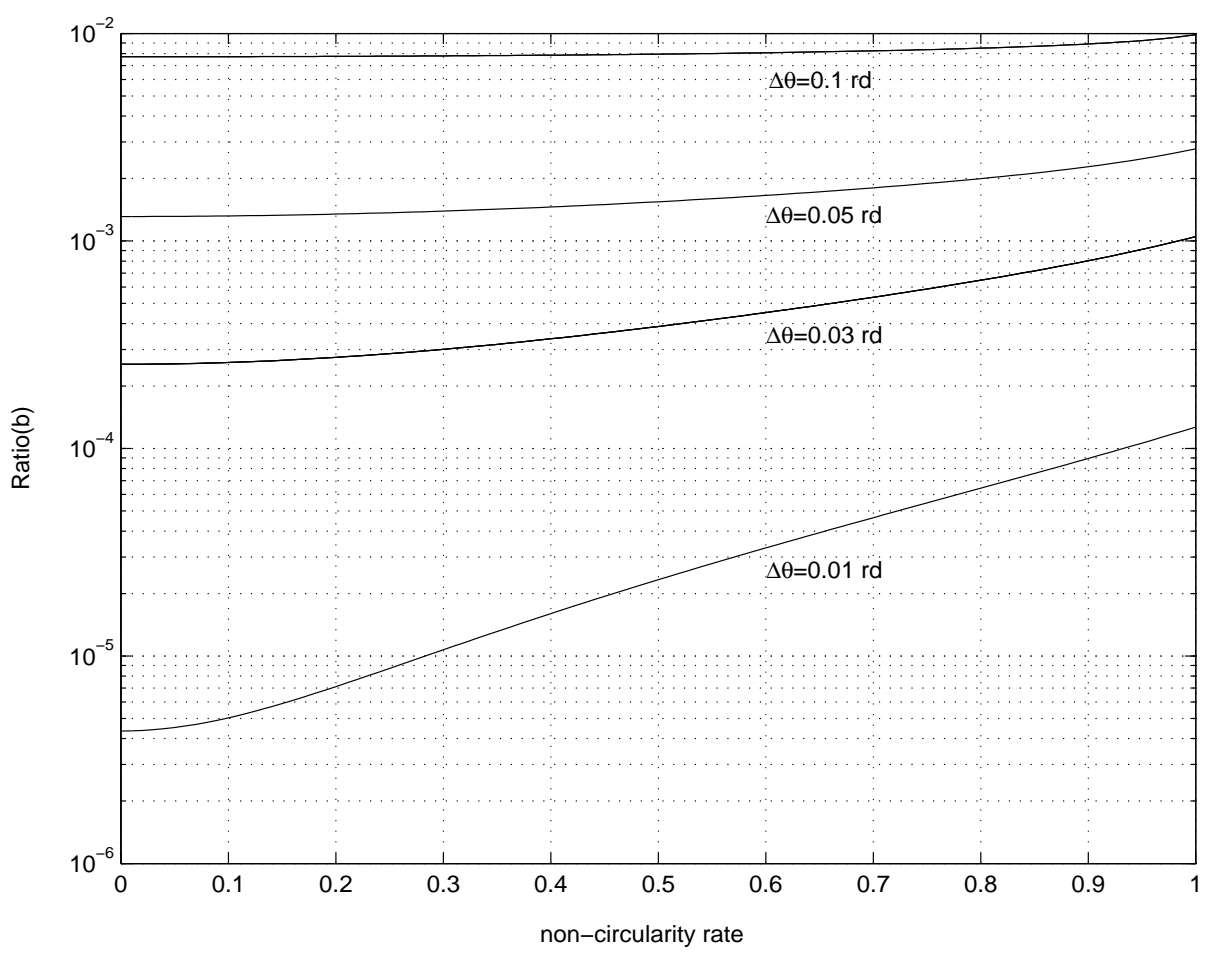

Fig.4 Ratio $r_{b} \stackrel{\text { def }}{=} \frac{\left(\mathbf{C}_{\Theta_{1}^{N C G}}\right)_{(1,1)}}{\left(\mathbf{C}_{\Theta_{1}^{N C G}}\right)_{(1,1)}}$ as a function of the non-circularity rate for different values of DOA separation $(\Delta \theta)$ for $\phi_{1}=\pi / 2$ and $\phi_{2}=\pi / 3$.

The second experiment illustrates result 5 where a non-circular complex Gaussian source and $M=3$ are considered. Fig. 5 shows that the CRB decreases monotonically as the non-circularity rate increases but it is relatively insensitive to the increase of $\rho_{1}$, except for very low SNR (i.e. for $\left\|\mathbf{a}_{1}\right\|^{2} r_{1} \approx 1$ ). 


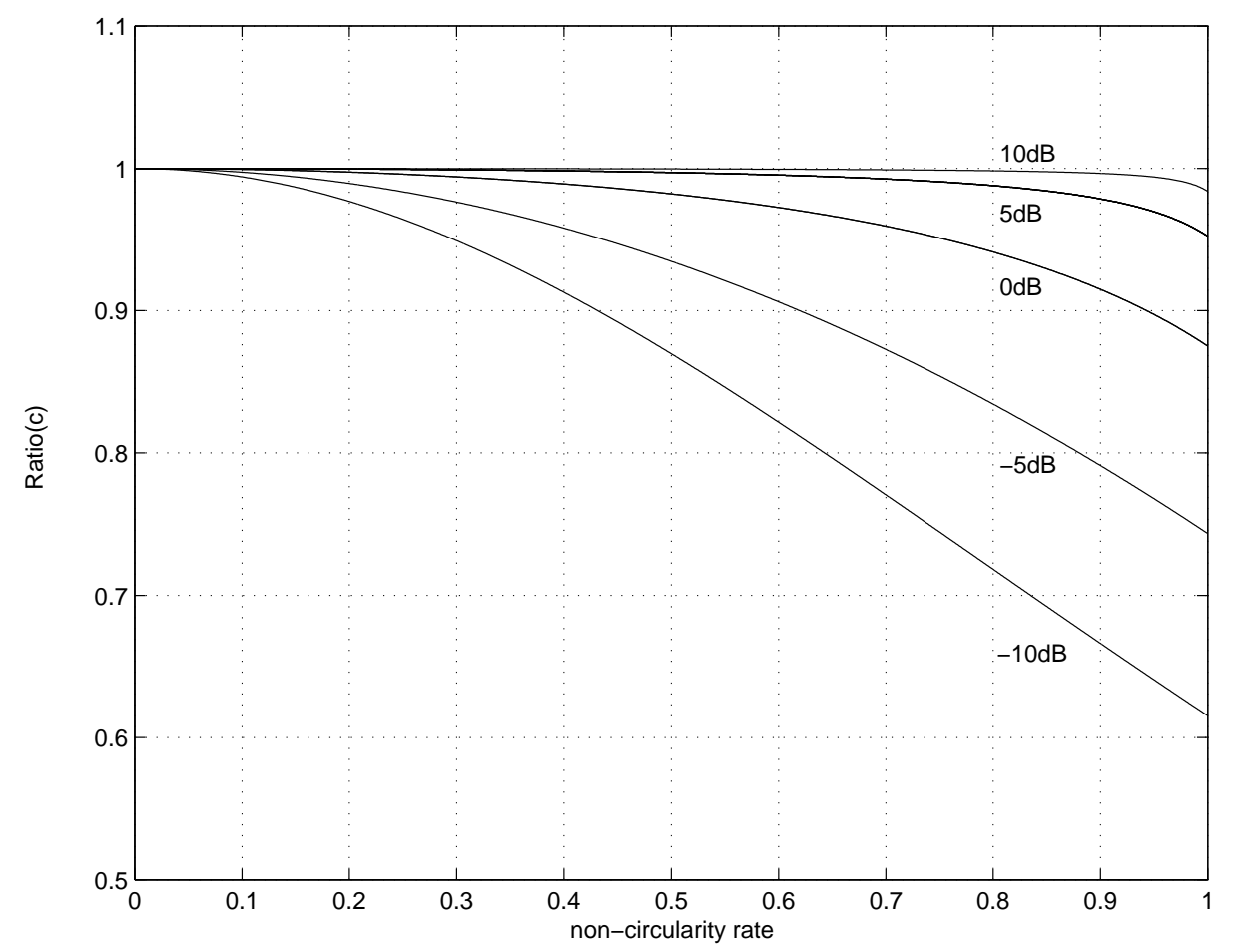

Fig.5 Ratio $r_{c} \stackrel{\text { def }}{=} \frac{\left(C_{\theta_{1}}^{\mathrm{NCG}}\right)}{\left(C_{\theta_{1}}^{\mathrm{CG}}\right)}$ as a function of the non-circularity rate $\rho_{1}$ for different values of the SNR $r_{1}$.

The two last experiments compare the numerical values of the non-circular complex Gaussian $\mathrm{CRB}_{\mathbf{\Theta}_{1}}^{\mathrm{NCG}}{ }^{\prime} 4$ with those of the CRB $\mathrm{C}_{\Theta_{1}}^{\mathrm{BPSK}}$ associated with several BPSK distributed sources. Because the associated PDF of $\mathbf{z}_{t}$ is a mixture of $2^{K}$ Gaussian PDFs, this latter CRB appears to be prohibitive to compute, we use a numerical approximation derived from the strong law of large numbers, i.e.

$$
\mathbf{C}_{\Theta_{1}}^{\mathrm{BPSK}}=\left(\mathbf{I}_{F}^{-1}\right)_{(1: K, 1: K)} \quad \text { with } \quad\left(\mathbf{I}_{F}\right)_{k, l}=\lim _{T \rightarrow \infty} \frac{1}{T} \sum_{t=1}^{T}\left(\frac{\partial \ln p\left(\mathbf{z}_{t} ; \Theta\right)}{\partial \theta_{k}}\right)\left(\frac{\partial \ln p\left(\mathbf{z}_{t} ; \Theta\right)}{\partial \theta_{l}}\right)
$$

where

$$
p\left(\mathbf{z}_{t} ; \Theta\right)=\frac{1}{2^{K} \pi^{M} \sigma_{n}^{2 M}} \sum_{l=0}^{2^{K}-1} e^{-\frac{\left\|\mathbf{z}_{t}-\mathbf{A}\left(\Theta_{1}\right) \mathbf{s}_{l}\right\|^{2}}{\sigma_{n}^{2}}} \text { with } \mathbf{s}_{l} \stackrel{\text { def }}{=}\left(a_{1} \epsilon_{1, l} e^{i \phi_{1}} \ldots, a_{K} \epsilon_{k, l} e^{i \phi_{K}}\right)^{T}
$$

where $\epsilon_{k, l}=-1,+1$ are given by the dyadic expansion $l=\sum_{k=1}^{K} \frac{\left(\epsilon_{k, l}+1\right)}{2} 2^{k-1}, l=0, \ldots, 2^{K}-1$, for $K$ independent unfiltered (i.e. $\left.\rho_{k}=1\right)$ BPSK sources for which $\Theta=\left(\Theta_{1}^{T}, \Theta_{2}^{T}\right)^{T}$ with here $\Theta_{2} \stackrel{\text { def }}{=}\left(\sigma_{1}^{2}, \ldots, \sigma_{K}^{2}, \phi_{1}, \ldots, \phi_{K}, \sigma_{n}^{2}\right)^{T}$.

In the third experiment, we consider the one source case where $M=3$. Fig. 6 compares $C_{\theta_{1}}^{\mathrm{NCG}}, C_{\theta_{1}}^{\mathrm{CG}}$ and $C_{\theta_{1}}^{\mathrm{BPSK}}$. This figure shows that the CRBs under the BPSK distribution and under the non-circular complex Gaussian distribution (with $\rho_{1}=1$ ) approximately coincide. They outperform the CRB under the circular complex Gaussian distribution for low SNR only.

\footnotetext{
${ }^{4}$ We note that in the one source case $\mathbf{C}_{\Theta_{1}}^{\mathrm{NCG}}$ and $\mathbf{C}_{\Theta_{1}}^{\mathrm{NCG}}$ coincide.
} 


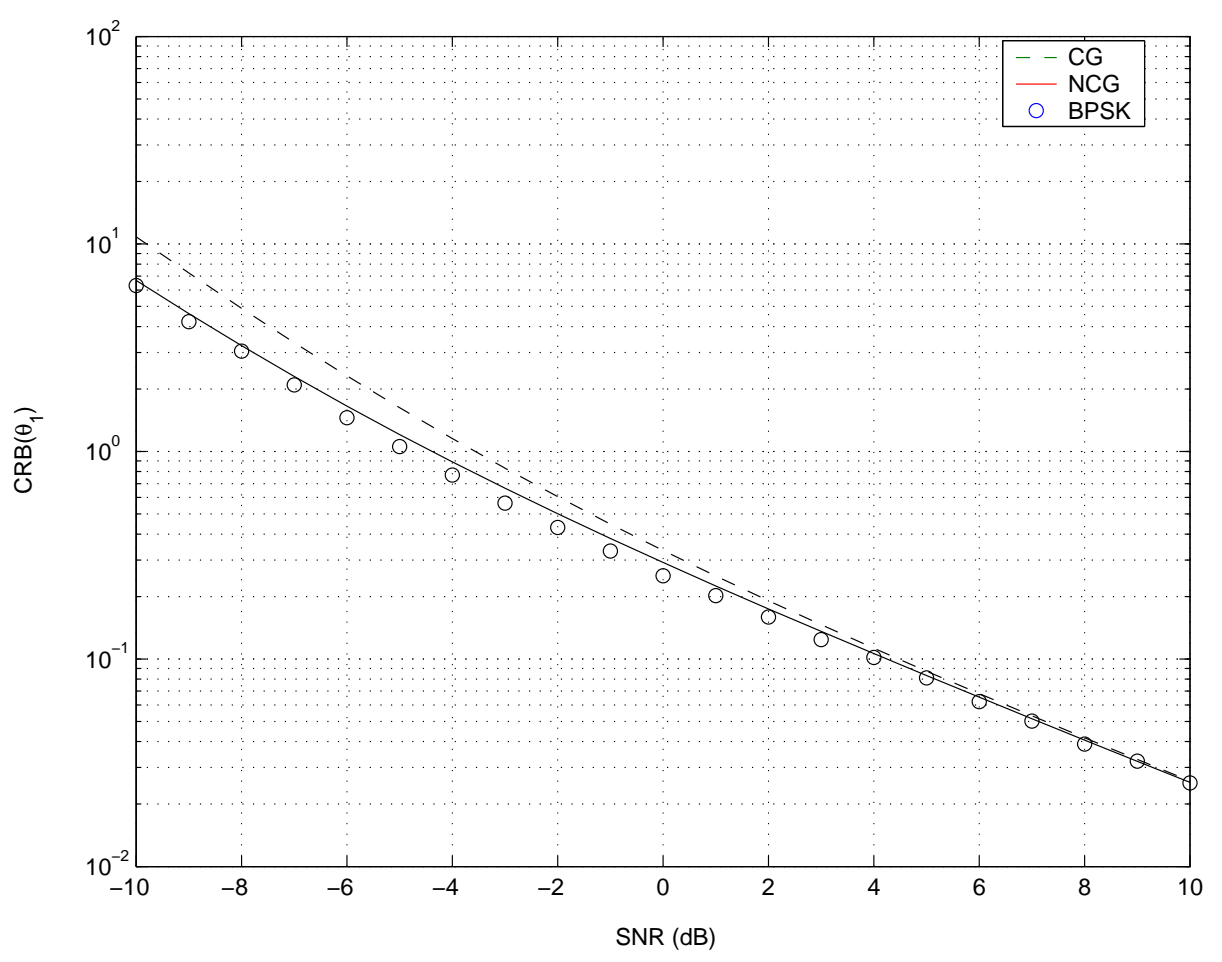

Fig.6 $C_{\theta_{1}}^{\mathrm{NCG}}, C_{\theta_{1}}^{\mathrm{CG}}$ and $C_{\theta_{1}}^{\mathrm{BPSK}}$ as a function of the SNR.

The last experiment illustrates the sensitivity of the CRB of the DOA to the distribution of the sources for two sources with $M=6$ when the a priori information that the two sources are independent are taken into account. Figs. 7,8 and 9 exhibit the difference between $\left(\mathbf{C}_{\Theta_{1}}^{\mathrm{NCG}^{\prime}}\right)_{(1,1)}{ }^{5}\left(\right.$ for $\left.\rho_{1}=\rho_{2}=1\right)$ and $\left(\mathbf{C}_{\Theta_{1}}^{\mathrm{BPSK}}\right)_{(1,1)}$ as a function of the SNR, the circularity phase separation $\phi_{2}-\phi_{1}$ and the DOA separation $\theta_{2}-\theta_{1}$, respectively. Fig. 7 shows that for the same a priori information, $\left(\mathbf{C}_{\Theta_{1}}^{\mathrm{BPSK}}\right)_{(1,1)}$ slightly outperforms $\left(\mathbf{C}_{\Theta_{1}}^{\mathrm{NCG}^{\prime}}\right)_{(1,1)}$ for all SNRs but tremendously outperforms the CRBs $\left(\mathbf{C}_{\Theta_{1}}^{\mathrm{CG}}\right)_{(1,1)}$ and $\left(\mathbf{C}_{\Theta_{1}}^{\mathrm{NCG}}\right)_{(1,1)}$ which do not take account of this a priori information. Figs. 8 and 9 show a weak sensitivity of $\left(\mathbf{C}_{\Theta_{1}}^{\text {BPSK }}\right)_{(1,1)}$ to the circularity phase separation $\phi_{2}-\phi_{1}$ and to the DOA separation $\theta_{2}-\theta_{1}$ w.r.t. $\left(\mathbf{C}_{\Theta_{1}}^{\mathrm{NCG}^{\prime}}\right)_{(1,1)}$ which is very sensitive. They also show that the difference between $\left(\mathbf{C}_{\Theta_{1}}^{\mathrm{NCG}^{\prime}}\right)_{(1,1)}($ for $\left.\rho_{1}=\rho_{2}=1\right)$ and $\left(\mathbf{C}_{\Theta_{1}}^{\text {BPSK }}\right)_{(1,1)}$ increases as the circularity phase separation or the DOA separation decreases.

\footnotetext{
${ }^{5}$ We note that comparing directly $\mathbf{C}_{\Theta}^{\mathrm{BPSK}}$ to $\mathbf{C}_{\Theta_{1}}^{\mathrm{NCG}}$ would be unfair because these CRBs are not associated with the same a priori information.
} 


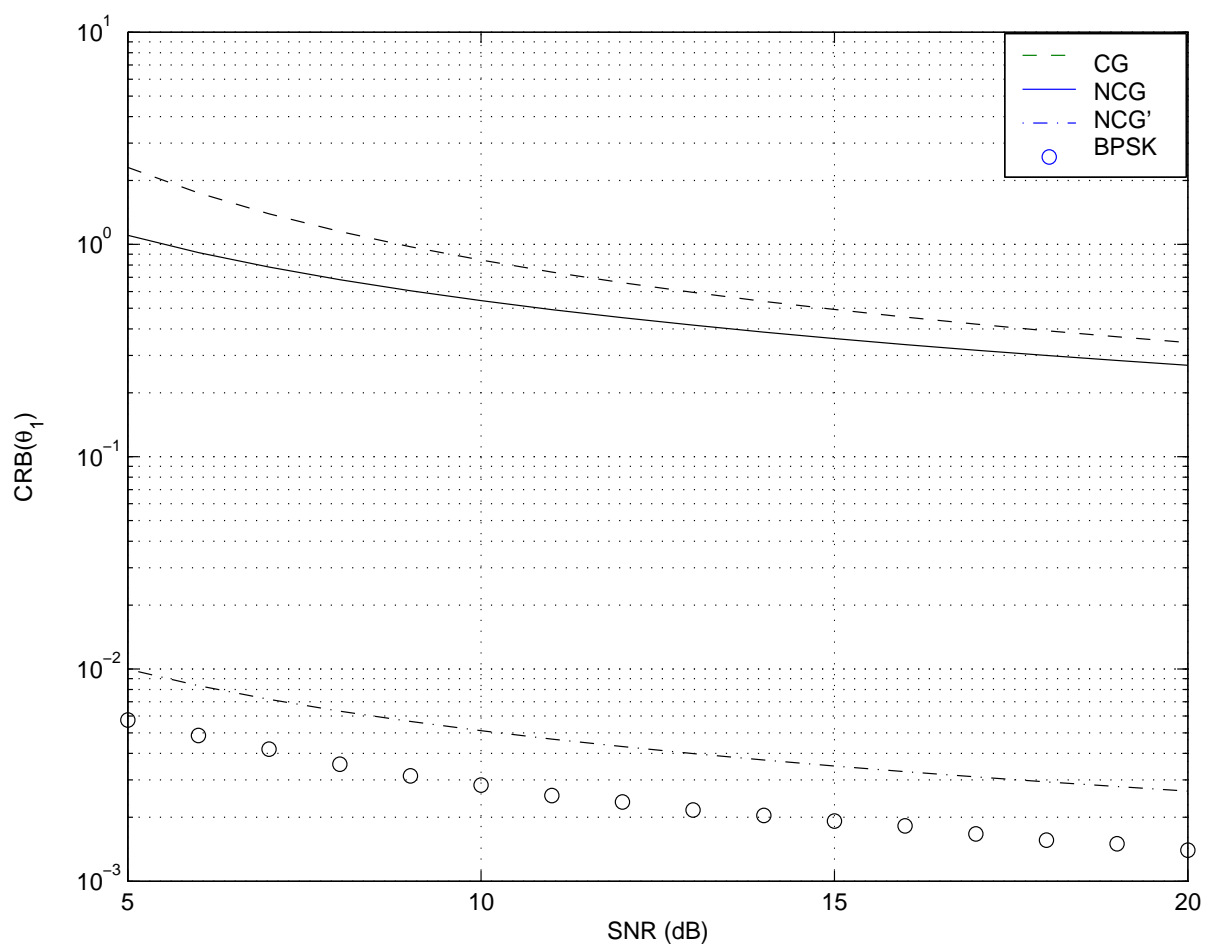

Fig.7 $\left(\mathbf{C}_{\Theta_{1}}^{\mathrm{BPSK}}\right)_{(1,1)}(\mathrm{o}$ o o $)$, and $\left(\mathbf{C}_{\Theta_{1}}^{\mathrm{NCG}}\right)_{(1,1)},\left(\mathbf{C}_{\Theta_{1}}^{\mathrm{NCG}}\right)_{(1,1)},\left(\mathbf{C}_{\Theta_{1}}^{\mathrm{CG}}\right)_{(1,1)}$ as a function of the SNR for a DOA separation of $0.1 r d, \rho_{1}=\rho_{2}=1$ and $\phi_{1}=\pi / 2, \phi_{2}=\pi / 3$.

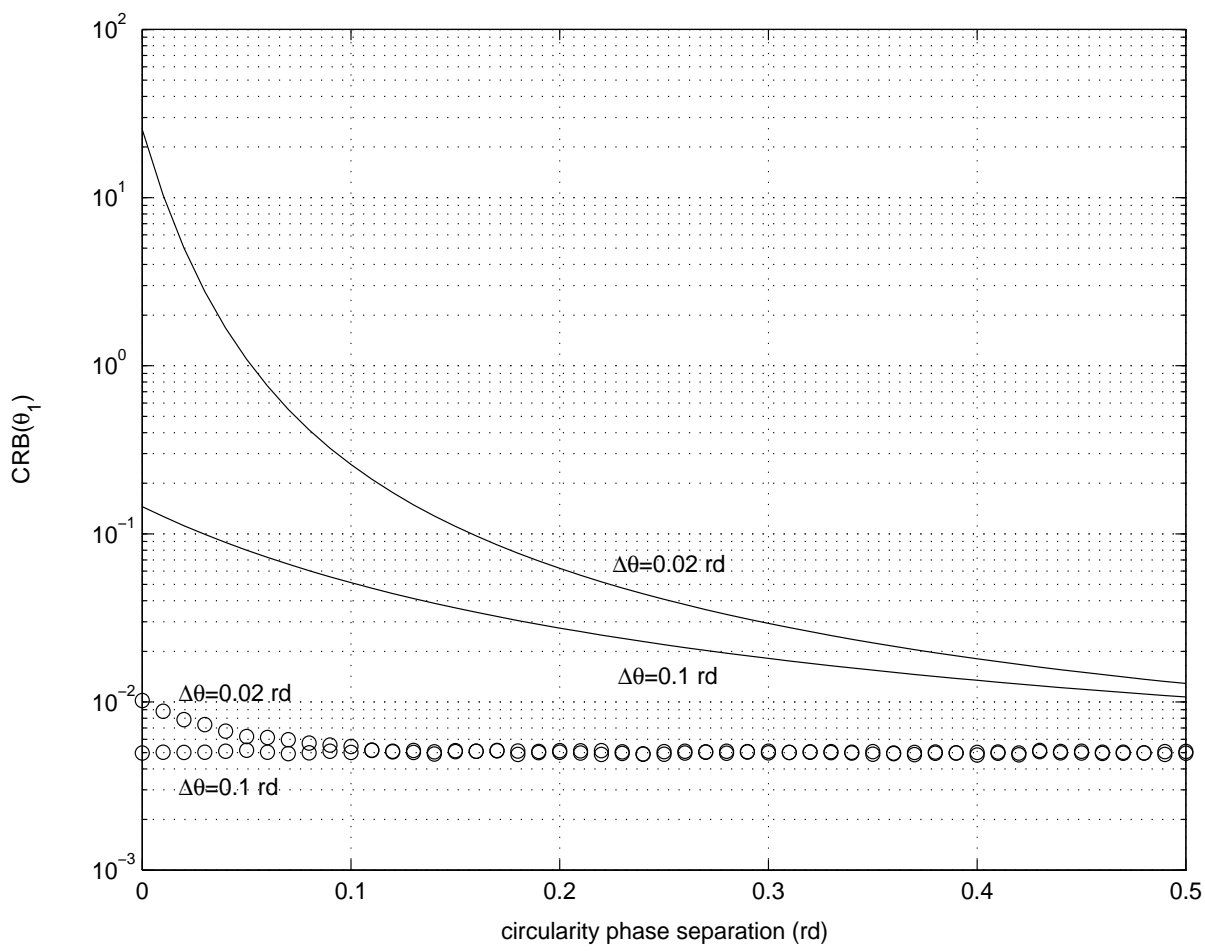

Fig.8 $\left(\mathbf{C}_{\Theta_{1}}^{\mathrm{BPSK}}\right)_{(1,1)}(\mathrm{o}$ o o $)$, and $\left(\mathbf{C}_{\Theta_{1}}^{\mathrm{NCG}}\right)_{(1,1)}$ as a function of the circularity phase separation for different values of DOA separation $(\Delta \theta)$ for $S N R=20 d B$. 


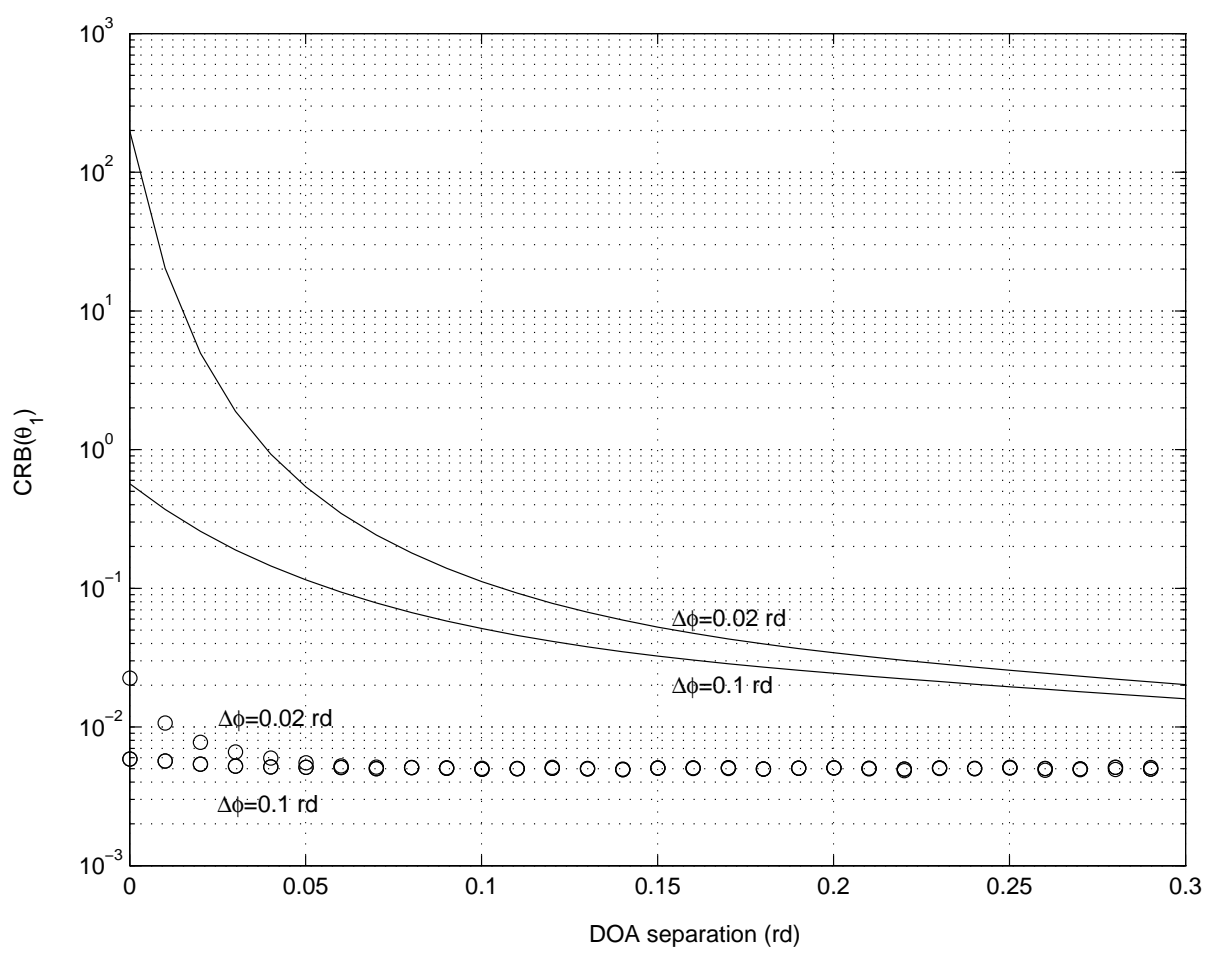

Fig.9 $\left(\mathbf{C}_{\Theta_{1}}^{\mathrm{BPSK}}\right)_{(1,1)}($ o o o $)$, and $\left(\mathbf{C}_{\Theta_{1}}^{\mathrm{NCG}^{\prime}}\right)_{(1,1)}$ as a function of the DOA separation for different values of circularity phase separation $(\Delta \phi)$ for $S N R=20 d B$.

\section{Conclusion}

This paper has provided an extension of the Slepian-Bangs formula for general non-circular complex Gaussian distributions then has focused on the stochastic CRB on DOA estimation accuracy for non-circular Gaussian sources. An explicit expression of the CRB for DOA parameters alone in the case of non-circular complex Gaussian sources by two different methods has been derived. Some properties of this CRB have been proved and finally, it has been numerically compared with the CRBs under BPSK distribution.

An issue which was not addressed in this paper is the stochastic CRB of BPSK or QPSK distributed sources and the comparison of these CRBs with those of the non-circular or circular complex Gaussian distribution. A paper has just been submitted to deal with this issue.

\section{A Appendix: Proof of (3.9)}

All the steps of the derivation of [7] apply with slight modifications. For the stochastic gradient and the deterministic hessian calculations, [7, rel. B(.16)] and [7, rel. B(.15)] apply where $\mathbf{A}$ and $\mathbf{R}_{z, T}$ are replaced respectively by $\tilde{\mathbf{A}}$ 
and $\mathbf{R}_{\tilde{z}, T}$. Using the partitioning of $\tilde{\mathbf{A}}, \boldsymbol{\Pi}_{\tilde{\mathbf{A}}}^{\perp}$ and $\mathbf{R}_{\tilde{z}, T}$, the expressions (3.11) and (3.12) follow.

For the derivation of (3.9) from (3.10), all the steps of [7] apply to prove that $\lim _{T \rightarrow \infty} \mathrm{E}\left(\left[\mathbf{F}_{T}^{\prime}\left(\Theta_{1}\right)\right]\left[\mathbf{F}_{T}^{\prime}\left(\Theta_{1}\right)\right]^{T}\right)=\mathbf{F}^{\prime \prime}\left(\Theta_{1}\right)$ except that here four terms are concerned from the expression of (3.11).

\section{B Appendix: Proof of the monotony of $C_{\theta_{1}}$ with $\rho_{1}$}

Because (3.14) may be written as the following function of $x \stackrel{\text { def }}{=}\left\|\mathbf{a}_{1}\right\|^{2} r_{1}$

$$
C_{\theta_{1}}^{\mathrm{NCG}}=\frac{\left\|\mathbf{a}_{1}\right\|^{2}}{\alpha_{1} c}\left(-1+\frac{a+b}{b+\rho_{1}^{2}}\right) \quad \text { with } \quad a \stackrel{\text { def }}{=} \frac{(1+x)^{2}}{x^{2}}, \quad b \stackrel{\text { def }}{=} \frac{1+x}{1-x} \text { and } c \stackrel{\text { def }}{=} 1-x
$$

and that $\frac{a+b}{c}=\frac{(1+x)}{x^{2}(1-x)^{2}}>0, C_{\theta_{1}}^{\mathrm{NCG}}$ is a decreasing function of $\rho_{1}$.

\section{Appendix: Indirect proof of (3.9)}

All the steps of the direct derivation of [5] apply where [5, rel. (15)] is replaced by

$$
\mathbf{R}_{s}=\left[\mathbf{c}_{1}, \ldots, \mathbf{c}_{K}\right]=\left[\begin{array}{c}
\mathbf{c}_{1}^{H} \\
\vdots \\
\mathbf{c}_{K}^{H}
\end{array}\right] \quad \text { and } \quad \mathbf{R}_{s}^{\prime}=\left[\mathbf{c}_{1}^{\prime}, \ldots, \mathbf{c}_{K}^{\prime}\right]=\left[\begin{array}{c}
\mathbf{c}_{1}^{\prime T} \\
\vdots \\
\mathbf{c}_{K}^{\prime T}
\end{array}\right]
$$

and $[5$, rels. $(16),(17),(18)]$ become respectively

$$
\frac{d \mathbf{R}_{\tilde{z}}}{d \theta_{k}}=\mathbf{D}_{k} \mathbf{C}_{k}^{H} \tilde{\mathbf{A}}^{H}+\tilde{\mathbf{A}} \mathbf{C}_{k} \mathbf{D}_{k}^{H} \text { with } \mathbf{D}_{k} \stackrel{\text { def }}{=}\left(\begin{array}{cc}
\mathbf{d}_{k} & \mathbf{0} \\
\mathbf{0} & \mathbf{d}_{k}^{*}
\end{array}\right), \quad \mathbf{d}_{k} \stackrel{\text { def }}{=} \frac{d \mathbf{a}_{k}}{d \theta_{k}} \text { and } \mathbf{C}_{k} \stackrel{\text { def }}{=}\left(\begin{array}{rr}
\mathbf{c}_{k} & \mathbf{c}_{k}^{\prime} \\
\mathbf{c}_{k}^{\prime *} & \mathbf{c}_{k}^{*}
\end{array}\right)
$$

and

$$
\mathbf{Z}_{k}=\mathbf{R}_{\tilde{z}}^{-1 / 2} \tilde{\mathbf{A}} \mathbf{C}_{k} \mathbf{D}_{k}^{H} \mathbf{R}_{\tilde{z}}^{-1 / 2}
$$

Consequently [5, rel. (30)] becomes

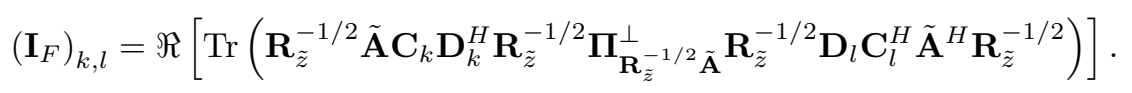


Then, thanks to $\mathbf{R}_{\tilde{z}}^{-1 / 2} \boldsymbol{\Pi}_{\mathbf{R}_{\tilde{z}}^{-1 / 2} \tilde{\mathbf{A}}}^{\perp} \mathbf{R}_{\tilde{z}}^{-1 / 2}=\frac{1}{\sigma_{n}^{2}} \Pi_{\tilde{\mathbf{A}}}^{\perp}=\frac{1}{\sigma_{n}^{2}}\left(\begin{array}{cc}\boldsymbol{\Pi}_{\mathbf{A}}^{\perp} & \mathbf{O} \\ \mathbf{O} & \boldsymbol{\Pi}_{\mathbf{A}^{*}}^{\perp}\end{array}\right)$, [5, rel. (32)] must be replaced by

$$
\begin{aligned}
\left(\mathbf{I}_{F}\right)_{k, l}=\frac{1}{\sigma_{n}^{2}} \Re[\operatorname{Tr} & \left(\left(\begin{array}{cc}
\mathbf{d}_{k}^{H} & \mathbf{O} \\
\mathbf{O} & \mathbf{d}_{k}^{T}
\end{array}\right)\left(\begin{array}{cc}
\mathbf{\Pi}_{\mathbf{A}}^{\perp} & \mathbf{O} \\
\mathbf{O} & \boldsymbol{\Pi}_{\mathbf{A}^{*}}^{\perp}
\end{array}\right)\left(\begin{array}{cc}
\mathbf{d}_{l} & \mathbf{O} \\
\mathbf{O} & \mathbf{d}_{l}^{*}
\end{array}\right)\right) \\
& \left.\left(\left(\begin{array}{cc}
\mathbf{c}_{l}^{H} & \mathbf{c}_{l}^{\prime T} \\
\mathbf{c}_{l}^{\prime H} & \mathbf{c}_{l}^{T}
\end{array}\right)\left(\begin{array}{cc}
\mathbf{A}^{H} & \mathbf{O} \\
\mathbf{O} & \mathbf{A}^{T}
\end{array}\right) \mathbf{R}_{\tilde{z}}^{-1}\left(\begin{array}{cc}
\mathbf{A} & \mathbf{O} \\
\mathbf{O} & \mathbf{A}^{*}
\end{array}\right)\left(\begin{array}{cc}
\mathbf{c}_{k} & \mathbf{c}_{k}^{\prime} \\
\mathbf{c}_{k}^{\prime *} & \mathbf{c}_{k}^{*}
\end{array}\right)\right)\right]
\end{aligned}
$$

Exploiting the structure $\left[\begin{array}{cc}\mathbf{G} & \mathbf{G}^{\prime} \\ \mathbf{G}^{*} & \mathbf{G}^{*}\end{array}\right]$ of $\mathbf{R}_{\tilde{z}}^{-1}$, relation (3.9) is straightforwardly deduced.

\section{References}

[1] D. Slepian, "Estimation of signal parameters in the presence of noise," in Trans. IRE Prof. Grop Inform. Theory PG IT-3, pp. 68-89, 1954.

[2] W.J. Bangs "Array processing with generalized beamformers," Ph.D. thesis Yale University, New Haven, CT, 1971.

[3] P. Stoica, R. Moses, "Introduction to spectral analysis," Prentice-Hall, Upper Saddle River, NJ, 1997.

[4] B. Picinbono, "Second-order complex random vectors and normal distributions," IEEE Trans. on Signal Processing, vol. 44, no. 10, pp. 2637-2640, October 1996.

[5] P. Stoica, A.G. Larsson and A.B. Gershman, "The stochastic CRB for array processing: a textbook derivation," IEEE Signal Processing letters, vol. 8, no. 5, pp. 148-150, May 2001.

[6] A.G. Jaffer, "Maximum likelihood direction finding of stochastic sources: a separable solution," in Proc. ICASSP New York, pp. 2893-2896, 11-14 April 1988.

[7] P. Stoica, A. Nehorai, "Performance study of conditional and unconditional direction of arrival estimation," IEEE Trans. on Acoustics Speech and Signal Processing, vol. 38, no. 10, pp. 1783-1795, Oct. 1990.

[8] M.G. Kendall, A. Stuart "The advanced theory of statistics," Hafner, New York 1961.

[9] P. Chargé, Y. Wang and J. Saillard, "A non-circular sources direction finding method using polynomial rooting," Signal Processing, vol. 81, pp. 1765-1770, 2001. 
[10] B. Ottersten, P. Stoica and R. Roy, "Covariance matching estimation techniques for array signal processing," Digital Signal Processing, vol. 8, pp. 185-210, July 1998.

[11] J.P. Delmas, "Asymptotically minimum variance second-order estimation for non-circular signal with application to DOA estimation," accepted for publication in IEEE Trans. on Signal Processing, 2003. 